LE MOIS HYDRO-ÉLECTRIQUE en France et a l'Etranger

\section{INFORMATIONS DIVERSES}

\section{Texte de l'exposé des motifs présentés à la Chambre des Députés, à l'appui du projet de loi sur les Dis- tributions d'énergie.}

\author{
RAPPORT DE W. GUILIAIN (suite el fin)
}

Art. 12.

Lorsiue le concessionnatre d'une distribution d'énergie contreLorstue le clauses du cahier des charges ou aux décisions rendues in exécution de ces clauses, en ce qui concerne le service de la naqigation ou des chemins de fer ou tramways, la ziabilité des voies mationales, départementales ou communales, ou le libre écoulement der caux, procès-rerbal sera dressé de la contrazention par les agents in service intéressé dûnent assermentés.

Ces contravontions seront poursuivies et jugées comme en matière de grande zoirie et punies d'une amende de Io à 300 trancs, sans préjudice de la réparation du dommage causé.

LiAdministration pourra d'ailleurs prendre immédiatement toute's les mesures provtsoires pour faire cesser le dommage comme ul cst procédè en matière de vourie. Les frais qu'entraînera l'exécution de cos mesures seront recouvrés contre le concessionnare comme en melière do contributions directes.

Ces dispositions sont la reproduction abrégée de celles du titre I: de la loi du 15 juillet 1845 sur la police des chemins de fer, avec cilto différence que, d'après Jadite loi, les contraventions sont punies d'ur'e amende de 300 à 3.000 francs. La Commission a pensé, avec le Gouver. nement qu'étant donné la moindre importance des ouvrages relatif́, au tansport de l'énergie, il convenait de s'en tenir à l'amende ordinaıre de 16 à 300 francs des contraventions de grande voirie.

\section{Art. 13.}

Toute infraction aux dispositions édictées dans l'intérêt de la sécuritú des personnes, soit par les règlements. d'administration publique, so't par les arrêtés du Ministre des Travaux publics ou des Préfets, pris pour l'exécution desdits règlements, sera poursuvie devant les tribunaux correctionnels et punie d'une amende de 16 à 3.000 francs, sans prépudice de l'applacation des pénalités préoues au Code pénal en cïs diacident résultant de l'infraction.

Tandis que l'article précédent visait seulement les infractions du concessionnaire susceptibles de porter atteinte à l'ntérêt public de la circulation, l'article 13 s'applique aux infractions qui pourraient être commises solt par les permissionnaires ou les concessionnaires des transports d'énergie, soit par des tiers, aux dispositions réglementaires édictées dans l'mntérêt de la sécurité des personnes. La peine de 16 à 3.000 francs d'amende prévue pour ce cas, est celle qui est prévue pour les cas analogues par l'article 2 I de la loi du 15 juillet 1845 sur la police des chemins de fer.

Cet article 2 r prévoit en outre, pour les chemins de fer, qu'en cas de récidive dans l'année, l'amende sera portée au double, et le tribunal pourra, selon les circonstances, prononcer en outre un emprisonnement de trois jours à un mois. Le Gouvernement et la Commission ont pensé qu'il serait peut-être excessif d'adopter ces pénalités pour les transports d'énergie, alors qu'il s'agit d'infractions qui ne peuvent causer que des accidents individuels, et non pas des catastrophes comme dans le cas des chemins de fer. Il nous a semblé suffisant de viser l'application normale du Code pénal en cas d'accident

Notre projet de loi passe sous silence les autres infractions aux reglements d'administration publique pris en vertu de l'article it ou aux arrêtés ministériels ou préfcctoraux renclus pour leur exécution. Pat suite ces infractions ne seront passibles que des peines de droit commun. La Commission estime qu'il n'y avait pas d'intérêt public à
aller au delà.

Il tésulte de l'article 13 que le Ministre des Travaux publics aura qualité pour régler dırectement, par voles d'arrêtés mınistériels, et non plus seulement par la vose indirecte d'arrêtés préfectoraux uniformes, les mesures de détail, communes à tout le territoire, relatives à l'exécution des règlements d'administration publique.

\section{Art. 14.}

Les délits et contraventions pourront être constatés par des procèsverbaux dressés par les officuers de police juduciaire, les ingéneurs ot agents des ponts et chaussies et des mmes, les agents voyers, les agents muncipaux chargés de la survellance ou du contrôle, et les gardes particuliers du concessionnaire agréés par lAdministration et dìment assermentés.

Ces procès-verbaux feront foi jusqu'à preuce du contraire.

Its seront visés pour timbre et enregistrés cn débet.

Ceux qui auront été dressés par des gardes assermentés devont citne affrmés dans les trois jours, à peine de nullité, devant le juge de part ou le maire, soit du lieu de délut ou de la contravention, soit de la résidence de l'agent.

Cet artıcle quı ne dıffère du texte du Couvernement que par l'additaon de quelques mots destinés à y apporter plus de précision, est nécessaire pour habiliter les fonctionnaires et agents clu contrôle, ceux de la voirie et les gardes particulicrs des concessionnaires à dresser des procès-verbaux farsant foi en justice.

\section{Art. I5.}

La déclaration d'utilité publique d'un chemin de fer, d'un tramsvay, d'une vore navigable ou en général d'un travanl publu confère à l'Administration ou au concessionnaure, pour l'établussement et le fonctionnement des conducteurs d'énergie employés à l'explontation de cês ouvrages, les drouts de passage et d'appun spécifiés aux articles 7 et 8 cr-dessus, avec application des dispositions des arlucies 9 et 10 et des dispositzons spéciales édactées à cet effet par les règlements d'admmistration publique prévus à l'article $I I$, même dans le cas où l'énergie seraut fournie à ces conducteurs par une usine priqée ou par une entreprise de distribution publique d'énergıe non déclarée d'utilité publique.

Toutefors, par dérogation au $\$ I^{0}$ do l'ant 7 , les conducteurs aériens on contact avec les organes de prise de courant de véhicules, et leurs jonctions avec les conducteurs d'almentation ne sont pas assujettis à être placés au-dessus des fenétres les plus élevées des habitations.

Le projet du Gouvernement ne visait que les distributions publiques d'énergie. Or le transport de l'énergie pour le service des tramways, des chemins de fer et des voies navigables, prend de jour en jour une importance croissante et, pour lc facliter, ll est nécessaure de conférer à l'administration et aux concessionnaires, pour le passage et l'appui de leurs conducteurs, les droits que la présente loi, en ses articles 7,8 , 9 et to confère aux distributions publiques d'énergie. Il arrive en effet, notamment pour les tramways, que l'installation du système si économique de traction électrique par fils aériens, dans certaines rues qui sont trop étroites pour se prêter à l'implantation de nombreux poteaux, est rendue presque impossible par la résistance des propriétaires qui refusent de laisser sceller dans les façades de leurs maisons les attaches des câbles transversaux soutenant les conducteurs aériens en contact avec les organes de prise de courant des véhicules.

La Commission considère qu'il est nécessarre de combler cette lacunc: de Ia Ioi, et c'est pourquoi elle propose le premier paragraphe de l'article 15.

Les règles de compétence des articles 9 et so s'appliqueront dans ce cas ; et les règlements d'administration publique prévus à l'article 11 pourront contenir des dispositions spéciales applicables à ce genre de transports d'énergie.

Les derniers mots du paragraphe ${ }^{\text {er }}$ de l'article 15 , "même dans le cas où l'énergie etc... ") ont pour but de fixer un point de jurisprudence administrative.

Les décrets de concession rendus en Conseil d'Etat ne s'étaient pas opposés à l'origine, à ce que les concessionnaires de tramways électriques eussent la faculté de prendre le courant électrique nécessaire à leur exploitation, à des usines qui ne feraient pas partie intégrante de la concession, et qui par suite ne devraicnt pas faire retour à l'autorité concédante, à l'expiration de la concession. C'est dans ce sens qu'ont été organisées un certain nombre d'entreprises. Les concessionnaires y trouvent l'avantagc de se procurer à meilleur compte l'énergie électrique, puisque les usines n'ont pas à être amortíes pendant le délai restreint de la concession. De plus, l'exploitation de 
ces usines restant libre, elles peuvent être employées, sans autorisations spéciales et sans avour à subir l'ingérance de l'administration, à fournır l'énergie électrıque à cles industriels, à des distributions municipales de lumière, etc. ; elles répartissent ainsi leurs frais généraux sur une plus grande masse d'affaires ; de là une réduction du prix de revient qui doit avoir sa répercussion sur les tarifs.

Mais, plus tard, l'Administration et le Conseil d'Etat ont craint sans doute que ce régıme de liberté ne laisse l'exploitation des tramways exposée à des interruptions fâcheuses, du fait de la négligence ou même de la défaillance des fournisseurs d'énergie qui échappent ainsi à l'action de l'autorité concédante. Et depuis quelque temps les décrets de concession de tramways électriques imposent toujours au concessıonnaire l'obligation d'avoir des immeubles affectés à la production d'électricité qui font partie intégrante de la concession et qui sont incorporés au domaine public.

Cettc exigence peut être justifiée lorsqu'ıl s'agıt d'une concession de tramways très étendue et à service très intensif. Elle serait certainèment excessive dans d'autres cas. Il convient de n'avoir pas de règle absolue en ces matières undustrielles. Aussi, le premier paragraphe de l'article 15 admet-il, in fine, que l'énergie nécessarre à l'exploitation des chemins de fer, tramways, voies navigables, etc., pourra leur être fourne par des usines particulières, sans priver les administrations ou les concssionnairs du bénéfice des droits d'appui et de passage poưr les conducteurs d'énergie spécialement affectés à leur service. İl résulte implicitement de cette disposition que les concessionnaires des tramways électriques pourront emprunter l'énergie nécessalre à leur service de traction à des usines quı ne seraient pas incorporées au domaine public, toutes les fois que le Gouvernement estimera qu'il n'y a pas nécessité de leur imposer cette incorporation en vue d'un intérêt public impérieux.

Le second paragraphe de l'article 15 a pour objet de tenir compte d'une nécessité technique des distributions d'énergie, spéciale aùx chemins de fer, tramways, etc., à traction électrique par fils aériens. Lorsque le véhıcule moteur ou automoteur doit prendre le courant électrique sur un conclucteur aérien, dit "de traction", au moyen d'un contact métallique placé à l'extrêmité d'un bras mobile fixé audit véhicule, on ne peut pas placer le conducteur aérien à plus de 5 à $6 \mathrm{~m}$. au-dessus du sol, sous peine de créer de sérieuses difficultés de fonctionnement. Or, d'après le paragraphe $\mathrm{I}^{\text {er }}$ de l'article 7 de notre projet de loi, les conducteurs aériens, dont les supports sont scellés d'office sur les immeubles privés, doivent être placés au-dessus des fenêtres les plus élevées. Il est nécessaire de spécifier, dans le cas particulier des " conducteurs de traction" de l'article I5, une exception à cette règle.

L'exception ne vise pas les "conducteurs d'alimentation" (ou feeders) qui amènent le courant électrique depuis l'usine jusqu'à proximité des " conducteurs de traction ". Ces conducteurs d'alimentation resteront soumis à toutes les règles de l'article 7. Mais il y aura nécessairement une exception pour les conducteurs secondaires qui établissent la jonction entre "les conclucteurs de traction" placés à 5 mètres ou 6 mètres au-dessus du sol, et " les conducteurs d'alimentation " placés au sommet des façades des maisons.

\section{RÉSUME}

En résumé, Messieurs, le projet de loi assure aux entreprises de distribution publique d'énergie la sécurité qui résulte d'un acte de concession, c'est-à-dire d'un contact conclu avec l'autorité publique, tout en leur permettant de fonctionner dans certains cas sous le régime plus libre, mais moins sûr, des simples permissions de voie précaires et révocables. Il organise virtuellement les entreprises concédées sous la forme de véritables services publics dont tous les habitants de leur ressort pourraient recueilır des avantages assurés. Il détermine les règles de compétence et les conditions d'ordre public relatives aux concessions. Il permet de conférer aux entreprises quı en seront jugées dignes, le caractère de travaux publics. Par la déclaration d'utilité publique, il donne à ces entreprises les moyens d'assurer le service public, malgré les résistances des intérêts particuliers. Enfin il confère aux distributions d'énergie employées au service des chemins de fer, tramways et autres ouvrages publics les mêmes ciruits qu'aux distributions publiques.

Ces dispositions, nous en sommes convaincus, auront pour effet de donner à l'inclustrie vationale un nouvel instrument d'une grande puissance. D'accord avec le Gouvernement ,nous vous demandons de les adopter en votant le projet de loi dont la teneur suit :

\section{PROJET DE LOI}

Article premier.

Les entreprises ayant pour objet le transport de l'énergie en vue d'en faire la distribution au public au moyen d'ouvr: ges fixes, sont soumises, pour leur établissement et leur fonctionnement, aux condi. tions de la présente loi.

\section{Art. 2.}

L'autorité compétente pour autoriser l'occupation d'une voie publique par les ouvrages d'une distribution d'éncrgie, peut se refuser à délivrer une simple permission de voirie, et subordonner l'occupation à une concession avec cahier des charges et tarif maximum.

La décision ainsi prise par le maire pour les voies publiques placées dans ses attributions peut être annulée, et la permission de voirie accordée par le Préfet dans les conditions prévues par l'art. 98 de la loi du 5 avril 1884 .

\section{Art. 3 .}

La concession d'une distribution publique d'énergie est donnée, après enquête, par la commune sj la distribution publique d'énergie ne dessert que son territoire, par l'Etat dans tons les autres cas.

Toute concession est soumise aux clauses d'un cahier des charges conforme à l'un des types approuvés par décret délibéré en Conseil d'Etat, sauf les dérogations ou modifications qui seraient expressément formulées dans les conventions passées au sujet de ladite conces. sion.

\section{Art. 4 .}

Lorsque la concession est de la compétence de l'Etat, l'acte de concession est passé définitivement par le Préfet, si la distribution d'énergie ne dessert que le territoire du département, ou par le Ministre des Travaux publics, après avis du Ministre de l'Intérieur, si elle dessert plusieurs départements.

Lorsque la concession est de la compétence de la commune, l'acte de concession passé par le Maire, en exécution d'une délibération du Conseil municipal est approuvé par le Préfet.

Toutefors, si l'acte de concession passé par le Ministre, le Préfet ou le Maire, comporte des dérogations ou modifications au cahier des charges type, il ne devient définitif qu'après avoir été approuvé par un décret délibéré en Conseil d'Etat.

\section{Art. 5.}

Aucune concession ne peut faire obstacle à ce qu'il soit accordé des permissions ou concessions concurrentes.

Toutefois, au cas de déclaration d'utilité. publique des travaux, l'autorité concédante peut s'interdire de donner des concessions ou des permissions de voirie pour des distributions de- même nature, pendant une durée ne pouvant excéder quinze ans à partir de l'expiration du délai fixé pour le commencement de la mise en exploitation.

L'acte de concession ne peut imposer au concessionnaire aucune charge pécuniaire autre que les redevances prévues à l'article $\mathrm{n}, \mathrm{ni}$ attrıbuer à l'Etat ou à la commune des avantages particuliers autres que les prix réduits d'abonnement qui seraient accordés aux services publics.

L'exécution des ouvrages destinés au transport et à la clistribution de l'énergie peut être cléclarée d'utilité publique, après enquête, par décret délibéré en Conseil d'Etat, sur le rapport des Ministres des Travaux publics et de l'Intérieur.

\section{Art. 7 .}

La déclaration d'utilité publique d'un transport d'énergı confère au concessionnaire, dans les conditions spécifiées par les règlements d'administration publique prévus à l'article I i et par le capier des charges de la concession, le droil :

$I^{\circ}$ D'établir à demeure des supports pour conducteurs aériens d'énergie, soit à l'extérieur des murs ou façades donnant sur la vóé publique, de manière que les conducteurs soient toujours placés atrdessus des fenêtres les plus élevées et hors de la portée des habitants, soit sur les toits et terrasses des bâtiments, à la condition qu'on puisse y accéder par l'extérieur ;

$2^{\circ}$ De faire passer des conducteurs d'énergic au-clessus des proptiètés privées, à la condition qu'ils soient hors de portée ; 
$3^{0}$ D'établir à demeure des canalisations souterraines ou des supports pout conductcurs aériens sur les terrains privés non bâtis qui ne sont pas formés de murs ou autres clôtures équivalentes.

Art. 8.

L'exécution des travaux prévus à l'article 7 doit être précédée d'une notification directe aux intéressés et d'une enquête spéciale dans chaque commune. Elle ne peut avoir lieu qu'après approbation des projets de délai du tracé par le Préfet.

Elle n'entraînè aucune dépossession. La pose d'appuis sur les murs ou façades ou sur les toits et terrasses des bâtiments ne peut faire obstacle au droit du propriétaire de démolir, réparer ou surélever. La pose de canalisations ou de supports dans un terrain ouvert et non bâti ne fat pas non plus obstacle au droit du propriétare de se clore ou de bâtir.

Mais le propriétaire devra, un mois avant d'entreprendre lcs travaux de démolition, réparation, surélévation, clôture de bâtıments, prévenur le conccssionnaire par lettre recommandée avec accusé de réception, adiessée au domicile élu par ledit concessionnaire.

Les indemnités qui pourraient être dues soit à raison des occupations et travaux prévus à l'article 7 , soit à raison des occupations temporaures de terrains que le concessionnaire seralt autorisé à pratiquer par application de la loi du 29 décembre 1892 , ou des dommages quelconques causés par l'exécution d'ouvrages déclarés d'utilite publique en vertu de la présente loi, sont réglées par le Conseil de préfecture, sauf recours au Conseil d'Etat.

Art. 10.

Iorsque pour l'établissement des ouvrages déclarés d'utilité publique, il y a lieu à expropriation, elle est prononcée et lcs indemnités sont réglées conformément à la loi du 3 mai $184 \mathrm{I}$.

Art. II.

Des règlements d'administration publique, rendus sur le rapport des Mimistres des Travaux publics et de l'Intérieur, détermineront :

$1^{0} \mathrm{La}$ forme des enquêtes prévues aux articles 3 , 6 et 8 ;

$2^{0}$ Les formes de l'instruction des projets et de leur approbation par l'autorité concédante, sans préjudice, quand il s'agit d'énergie électrique, de l'approbation des projets par le Ministre des Postes et des Télégraphes ou son délégué, au point de vue de la protection des tansmissions télégraphiques et téléphoniques, en vertu de l'article 5 de la loi du 25 juin 1895 ; les formes de l'homologation des tarifs par l'autorité concédante, et l'organisation du contrôle, dont les frais seront à la charge du concessionnaire ;

$3^{0}$ Les conditions générales et d'intérêt public auxquelles les ouvrages servant au transport et à la distribution de l'énergie, soit en vertu de concessions, soit en vertu de simples permissions de voirie, devront satisfaire tant pour leur construction que pour leur fonctionnement ;

$4^{\natural}$ Les mesures relatives à la police et à la sécurité de l'exploitation des transports et distributions d'énergie ;

$5^{\circ}$ Les tarifs des redevances dues à l'Etat, aux départements et aux communes en raison de l'occupation du domaine public par les ouvrages des entreprises concédées ;

$6^{\circ}$ 'Et en général toutes les mesures nécessaires à l'exécution do la présente loi.

Les règlements visés par les paragraphes $3^{\circ}$ et $4^{\circ}$ ci-dessus seront pris sur l'avis technique du Comité d'électricité institué par l'article 6 de la loi du 25 juin 1895 .

Art. 12.

Lorsque le concessionnaire d'une distribution d'énergie contreviendra aux clauses du cahier des charges ou aux décisions rendues en exécution de ces clauses, en ce qui concerne le service de la navigation ou des chemins de fer ou tramways, la viabilité des voies nationales, départementales ou communales, ou le libre écoulement des eaux, procès-verbal sera dressé de la contravention par les agents du service intércssé dûment assermentés.

Ces contraventions seront poursuivies et jugées comme en matière de grande voirie et punies d'une amende de 16 à 300 francs, sans préjudice de la réparation du dommage causé.

LAdministration pourra d'ailleurs prendre immédiatement toutes les

mesures provisoires pour faire cesser le dommage comme il est procédé

en matière de voiric. Les frais qu'entraînera l'exécution cle ces mesures seront recouvrés contre le concessionnaire comme en matières de contributions directes.

$$
\text { Art. } 13 .
$$

Toute infraction aux dispositions édictécs dans l'intérêt de la bécurité des personnes, soit par les règlements d'administration publique, soit par les arrêtés du Ministre des Travaux publics ou des préfets, pris pour l'exécution desdits règlements, sera poursuivie devant les tribunaux correctionnels et punie d'unc amende de 10 at 3.000 francs, sans préjudice de l'application des pénalités prévues au Code pénal en cas d'accident résultant de l'mnfraction.

Art. I4.

Les délits ct contraventions pourront être constatés par des procèsverbaux dressés par les officiers de police judiciaire, les ingénicurs ct agents des ponts et chaussées et des mincs, les agents voyers, les agents municipaux chargés de la survcillance ou du contrôle, et les gardes particulicrs du concessionnairc agréés par l'Administration et dûment assermentés.

Ces procès-verbaux feront foi jusqu'à preuve du contrairc.

Ils seront visés pour tımbre et enregistrés en débet.

Ceux qui auront été dressés par des gardes assermentés devront être affirmés dans les trois jours, à peine de nullité, clevant le juge de paix ou le maire, soit du lieu du délit ou de la contravention, soit de la rẹ́sidence de l'agent.

$$
\text { Art. } 15 \text {. }
$$

La déclaration d'utilité publique d'un chemin de fer, d'un tramway, d'une vole navigable ou en génćral d'un travail public, confère à l'Administration ou au concessionnairc, pour l'établussement et le fonctionnement des conducteurs d'énergie employés à l'exploitation de ces ouvrages, les droits de passage et d'appui spécifiés aux articles 7 ct 8 c1-dessus, avec application des dispositions des articles 9 et 10 'et des dıspositıons spéciales édictées à cet effet par les règlements d'administration publique prévus à l'article $\mathrm{I} I$, même dans le cas où l'énergic sẹrait fournic à ces conducteurs par une usine privée ou par une entreprise de distribution publique d'énergie non déclarée d'utilité publique.

Toutefois par dérogation au paragraphe $\mathrm{I}^{\circ}$ de l'article 7 , les conducteurs aériens en contact avec les organes de prise de courant des véhiculcs, et leurs jonctions avec les conducteurs d'alimentation ne sont pas assujettis à être placés au-dessus des fencttres les plus élevées des babitations.

\section{Art. 16.}

Sont abrogées toutes dispositions de lois ou règlcments contraires à celles de la présente loi.

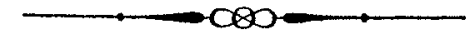

\section{ACADÉMIE DES SCIENCES}

\author{
Séance du 20 juillet 1903.
}

Sur la loi de recombinaison des ions. - Note de M. P. LANGEVIN.

Les gaz rendus conducteurs de l'électricité justifient, par toutes leurs propriétés, l'hypothèse que les charges disponibles y sont portées par un nombre infinı de centres électrisés ou ions, les uns positifs, les autres négatifs, ayant tous une mème charge égale en valeur absolue à celle que transporte un atôme monovalent dans l'électrolyse.

Ces ions participent au mouvement général d'agitation thermique des molécules du gaz, et le déplacement moyen de chacun d'eux, nul en l'absence d'un champ électrique extérieur, s'effectue dans le champ $X$ avee la vitesse $l_{1} X$ dans le sens des lignes de force pour les ions positifs, et $k_{2} \mathrm{X}$ dans le sens opposé pour les ions négatıfs. Les coefficients de proportionnalité $k_{1}$ et $k_{2}$ sont les mobilités des ions des deux signes.

L'attraction mutuelle des ions de signes contraires provoque une recombinaison progressıve des charges qu'ils transportent. L'expérience vérifie que, conformément aux prévisions théoriques, le rapport du nombre des recombinaisons au nombre des collisions entre deux ions de signes contraires, est toujours plus petit que l'unité et tend vers cette valeur quand. la pression du gaz augmente.

M. Langevin a pu, en suivànt de plus près, du point de vue 
cinétique, le mécanisme de la collision, montrer que ce rapport, aux faibles pressions, parie proportionnellement au carré de la pression.

La collision proprement dite entre deux ions de signes contraires commence au moment où, après un dernier choc contre les molécules neutres du gaz, les deux ions eni présence peuvent graviter librement l'un autour de l'autre en décrivant des coniques. L'auteur admet alors, pour démontrer cette loi, que la recombinatson a lieu lorsque, dans leur mouvement relatif, les centrés des deux ions, au moment du périhélie, se trouvent à une distance inférieure à une quantité donnée. Et la loi déduite du calcul est d'accord avec des fults d'expérience qui la confirment.

Essais sur la commutation dans les dynamos à courant continu. - Note de M. Iliovici.

« Dans l'étude expérimentale de la commutation il est intéressant d'étudier les questions suivantes :

" $1^{*}$ Variation de l'intensité du courant dans la section en courtcircuit, pendant la durée de la commutation;

" $2^{\circ}$ Variation de la force électromotriee indulte dans la section;

" 30 Variation de la chute de tension entre un balai et une lame du collecteur, nendant le temps où la lame touche le balai;

" $4^{0}$ Variation de la chute de tension entre un balat et le collecteur, le long du balai, pour une position déterminée du collecteur (et cela pour plusieurs positions de celui-c1).

(Ces questions doivent être étuliées pour divers régimes de fonctionnement de la dynamo : en fassant varier la vitesse de rotation, la position et la pression des balais, l'excitation, l'intensité du courant extérieưr, etc.

"Les expériences ont été faites sur une dynamo tétrapolarre de $20 \mathrm{kw} ., 110$ volts, $900 \mathrm{t} / \mathrm{m}$., enroulement tambour imbriqué; un balai couvrant deux lames $\gg$.

Io L’auteur a indiqué, dans une note précédente (séance du 22 juin Igo3), deux méthodes pour la mesure de l'mntensité.

$2^{\circ}$ "Pour étudier la variation de la force électromotrice induite dans la section étudiée, je relève, par la méthode Joubert, la force électromotrice induite dans la bobine de fil fin dont jas parlé dans la note citée. C'est très approximativement la force électromotrice induite dans la bobine de l'induit.

“ Il résulterait des courbes obtenues que le terme $L \frac{d i}{d t}+\Sigma M \frac{d t_{\mathrm{k}}}{d t}$ qui provient du flux de self-induction et d'induction mutuelle, joue un rôle prépondérant, lorsque la dynamo est parcourue par un fort courant.

$3^{\circ}$ " Pour étudier la variation de la chute de tension entre "un balai et une lame du collecteur, on relie la lame à une bague sur laquelle frotte un balai, et l'on relève par la méthode Joubert la courbe de différence de potentiel entre ce balai et le point du balai de la machine le plus rapproché du milieu de la lame considérée.

"J'ai obtenu des courbes pour diverses positions des balas. Lorsque les balais sont dans la ligne neutre, en fassant varier l'intensité du courant dans la machine, la chute de tension, qui ne varie pas beaucoup pour les positions pour lesquelles la lame est couverte en entier par le balai, augmente rapidement à la sortie de la lame de sous le balai, avec l'augmentation du courant, éest-à-dire avec la tendance à la production d'étincelles.

“ En déplaçant les balais, les chutes de tension à l'entrée et à la sortıe varient dans le sens contraire: la chute de tensıon à la sortie dıminue lorsqu'on avance les balais dans le sens de rotation de la machine. Le contraire arrive lorsqu'on déplace les balais dans le sens contraire.

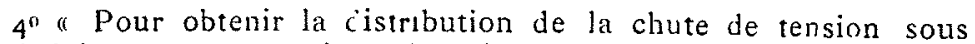
le balai pour une pusition déterminée du collecteur, on emploie la méthode suivante :

"Un petit balai très mince est monté sur une couronne graduée et frotte sur le collecteur. Ce balai et le point du balai de la machine le plus rapproché de son point de contact avec le collecteur sont réunis aux balass d'un contact tournant. Si l'on donne à ces balais une position fixe et que l'on place la balas auxillaire le long du collecteur en face du balai de la machine, on relève, à l'aide du contact tournant, la courbe de la chute de tension sous le balai pour une position déterminée du collecteur.

"En donnant aux balais du contact tournant une série de positions, on obtient une série de courbes qui nous montrent la distribution de la chute de tension entre balai et collecteur pour une série de positions de celui-ci. Pour les positions intermédiaires, on obtient les courbes par interpolation.

« Ceci nous donne encore une méthode pour l'étude de la variation de l'intensité du courant dans une spire en court-circuit, méthode plus longue et moins précise que les précédentes, mais qui a l'avantage de la simplicité du montage. De plus, elle nous donne l'intensité du courant au même instant dans toutes les bobines count. circuitées en même temps ». Et l'auteur termine sa communication en donnant la démonstration analytuque de ce fait.

Sur l'application de la fluorescéine à l'hydrologie souterraine. - Note de M. E.-A. Martel.

"L'emploi de la fluorescéine pour la recherche des relations entre les pertes et les réapparitions de rivières (imägı́é par Ten Brink en 1877 et de plus en plus généralisé) a donné lieu, récem. ment, à divers Mémoires ou Notes, dont certaines conclusions me paraissent tout au moins prématurées et établissent, en tout cas, que ce sujet est très insuffisamment élucidé. Comme je l'étudie moji. même assidûment depurs 1896 , aussi bien à l'air libre que sous terre, en examinant la marche des eaux intéricures parni les obstacles qu'elles rencontrent, je demanderaj la permission de fixer un peu les idées, en résumant très sommairement ce qui paraît acquis actuellement sur la question :

" $1^{\circ}$ La solution de fluorescérne, même tres concentrée, se décolore au soleil en moins de 24 heures.

" $2^{\circ} \mathrm{Au} \frac{\mathrm{r}}{20000000}$, en plein jour, mais à l'ombre, elle ne com mence à se décolorer qu'au bout d'une semaine au mons.

a 30 Dans l'obscurité complète je conserve, depuis 1897, des échantillons de solutions absolument inaltérées.

" $4^{\circ}$ La décoloration partielle par l'argile, reconnue par M. Trillat (Comptes-rendus, 13 mars 1899) est moindre sous pression qu' l'aır libre, remarque importante, puisque j'ai montré que, dans les réservoirs des cavernes, l'eau peut atteindre plusieurs atmosphères de pression (Comptes-rendus, 28 décembre 1896 ).

" 50 La Huorescéine, même dans une eau très chargée d'argile, ne se décante pas, contrairement à ce qui a été admis jusqu'ici au jour sans soleil (yoir $2^{\circ}$ ) elle se décolore lentement, sans étr entrainee par l'argile qui se dépose au fond du rase d'essai.

" 60 La coloration n'est modifiée ni par le filtre en papier, ni par la.bougie du filtre Chamberland, système Pasteur.

" $7^{\circ}$ La vitesse de propagation souterraine peut varier dans la proportion de I à 200 au moins; j'ai constaté 5 m50 à l'heure à Padirac (mai I 903 ) et $1050 \mathrm{~m}$ à l'heure à Bramabiau (septembre I 897 ) so:t $132^{\mathrm{m}}$ à $25 \mathrm{~km}$ par jour. Les causes de ralentissement dans l'écoth lement des eaux souterraines sont les éboulements rocheux, amas. de sable ou d'arglle, rétrécissements, siphonnements ou conduites forcées, expansions en bassins; bref, toutes les diminutions de section, multiplications de frottement et stagnations.

" 80 L'accélération est produite par l'augmentation de la pente et surtout par celle du debit (vitesse de Bramabiau, $5 \mathrm{~m}_{1} 8$ par minute, avec débit inférieur à $I^{\mathrm{m}^{3}}$ par seconde, et $\mathrm{I} 7^{\mathrm{m} 50}$ par munute avec $3 \mathrm{~m}^{3}$ par seconde; vitesse du Rhin à Ragaz, 8hm par heure pour $200^{\mathrm{m}^{3}}$ par seconde et 4 pour 1000 de pente; vitesse de la Tamina à Ragaz, 3hn par heure, débıt $9^{m m^{3}}$ par seconde et 50 pour 1000 de pente ; le torrent coule moins nite que le fleuve (août 1900).

" $9^{\circ}$ Les expériences de Ten Brink et Knop (1877) au Danube. Aach et de MM. Miquel et Dienert (Rapports de la Commission de Montsouris sur les sources du bassin de la Seine, 1901-1903) ont fournı des vitesses égales à celles de la fluorescéine, pour les disso* lutions de sel et la levure de bière (et même parfois inférieures).

" Io" La remise en marche de colorations souterraines est bien l'œuvre des crues internes; mais, en raison de l'absence de décantation ( 50 et $17^{\circ}$ ), on ne doit pas se prononcer encore sur la taçon dont elle se réalise; je pense qu'elle provient d'une baisse quir, après le jet de la fluorescéine, arrête l'eau colorée de bassins de retenue, momentanément privés d'écoulement, puis reınis en moly vement (vorre même anastomosés) par une chasse de crue. Elle peưt aussi être fictıve, par subdivisions inégales du cours souterrain.

" 1 10 Les eaux troubles des torrents glacidires et des cruss diminuent, jusqu'à l'annulation complète, la coloration même très forte.

« $12^{\circ}$ Mais l'expérimentateur peut remédier à cela par la décan: tation ou le filtrage des particules argileuses (\$50 et 60 ).

« i 30 Il est exact que la propagation de la fluorescéine semble moins rapide que celle de l'eau qui la véhicule; en eau très calmt j'ai trouvé, à Padirac (22 mai 1903), une vitesse de $12^{\mathrm{m}}$ par heure pour la tête d'une coloration et de $4^{\mathrm{m}}$ seulement pour la quelle, soit un retard des $2 / 3$ pour la fin de la couleur. En espaçantles jets de couleur, les derniers finissent toujours par rejondre les premiers.

« Mais il pourrait bien y avoir là (le fait étant contaire aus conséquences physiques de la parfaite incorporation moléculare 
de la fluorescéine dans l'eau, $\$ 50$ et $6^{\circ}$ ) une illusion, produite par de la fluor insuffisante des conditions matérielles de l'écoulement, une notion insuffariations incessantes de vitesse causées par les et surtes rencontrés. Ici, surtout, la circonspection s'impose.

En tout cas, ce retard, s'il est réel, et surtout les risques de retenue dans largile $\left(\$ 4^{\circ}\right)$ qui peuvent aboutir à la dilution et à rinvistbulité complètes, en cas de très long ou de très lent parcours, permettent de dire qu'un résultat négatif tiendra, le plus souvent, pera trop petite quantité de fluorescéine employée. Quel que soit linconvénient d'un excès de coloration, c'est le seul moyen de compenser les nombreux éléments d'insuccès des expériences. Et to précicux fluorescope de MM. Trillat et Marboutin doit être te précien plus comme correctif de ces eléments que comme moyen d'épargner la substance ou d'en restreindre les effets.

150 Il faut jeter la fluorescéine, non pas lentement et par petites " t ${ }^{\circ}$ '́ à la fois, mais, au contraire, rapidement et abondamment, afin de commencer toute expérience avec le maximum possible de afin de comn

" 160 Labsence de décantation se manifeste aussi sous terre : à padirac, du 23 mai au 7 juin 1903 , avec 750 de fluorescéine, j'di Padrac, coloré, pendant is jours, un bassin de $5000^{3} \mathrm{~m}^{3} .600 \mathrm{~m}^{3}$, no quacune trace de couleur soit demeurée ensuite perceptible a l'oel nu), même sous $5^{\text {nr }}$ de profondeur; la décoloration a été lente et progressive à partir du troisieme jour.

lente et progropation de la couleur en eau très calme se fait en inces filaments vasculaires, à la surface ou entre deux eaux, mais sans chute vers le fond $\left(\$ 50\right.$ et $\left.17^{\circ}\right)$.

180 Toute expérience devrait être faite de préférence lors des crues et même dans les trois états d'eaux basses, moyennes et hautes, les différences de résultats devant être émınemment instructives $₫$.

\section{Séance du 27 juillet igo3.}

Préparation et propriétés d'un siliciure de ruthénium. Note de MM. Henri Moissan et Wilhem Manchot.

Les auteurs indiquent qu'ils ont poursuivi ces recherches au moyen d'un bel échantillon de ruthénium métallique qui leur a été remis par M. Mathey, de Londres, et ils adressent tous leurs remerciements a ce grand industriel.

Lorsqu'on chaufte, disent-ils, au four électrique un mélange de 1 gr. 5 de ruthénium en poudre et de 7 grammes de silicium cristallisé, placé dans une nacelle de charbon au milieu d'un tube de même substance, on obtient rapidement la fusion du mélange, puss la combinaison se produit; elle est accusée par un rapide dégagement de vapeur, enfin le liquide revient tranquille et, à ce moment, on artête l'expérience. Cette dernière ne demande pas plus de deux a trois minutes avec un courant de 600 ampères sous 120 volts.

On obtient ainsi un culot métallique bien fondu et qui présente toujours le même aspect lorsqu'on fait varier le poids du silicium du simple au double. Cette substance est concassée, réduite en poudre, puss trattée par une lessive de soude au bain-marie et, ensuite, par un mélange d'acide fluorhydrique et d'acide nitrique. Il reste, après ces traitements, des cristaux blancs, brillants, mélangés à des quantités variables de carborundum que l'on peut sépater, grâce à leur différence de densité, au moyen de l'iodure de méthylène.

La densité de ce siliciure de ruthénium est de 5,40 à $4^{\circ} \mathrm{C}$. Il raye avec faclité le cristal de roche, la topaze et le rubis.Il est volatil au four électrique.

C'est un composé très stable; le fluor l'attaque cependant à froid et le chlore au rouge. Brusquement chauffé 11 brûle dans l'oxygène avec une belle incandescence. II n'est pas attaqué par tous les acides à leur température d'ébullition et le mélange d'acide nitrique et d'acide fluorhydrique, qui ne fournit aucune action à froid, ne réagit à chaud qu'avec une extrême lenteur. L'hypochlorite de potassium, qui attaque avec tant de facilité le ruthénium, est sans action sur ce sticiure.

Sa composition, répondant à la formule $R u S_{\imath}$ serait de: Ruthénium 78,17 , Silicium 21,83 .

\section{SOCIÉTÉ INTERNATIONALE DES ÉLECTRICIENS}

$$
\text { Séance du } I^{\text {er }} \text { juillet } 1903 .
$$

Résultats d'essaiş récents effectués sur les moteurs Couffinhal, par M. J. Courbier.
Jusqu'à présent, la variation de vitesse des moteurs à courant continu était obterue par l'introduction de résistances dans le circuit inducteur. On arrivait ainsı à avoir des vitesses dans le rapport extrème de 1 à 3 . Or, pour la commande des machines-outils, par exemple, il faut pouvolr disposer de vitesses variant dans des Imites plus étendues et, pour arriver à ce résultat, on se contentait habituellement d'un jeu de foulies sur lesquelles on faisait déplacer la courroie de commande et, même, lorsque la gamme des vitesses atnsi obtenues n'étalt pas suffisante, on avait recours à divers relats d'engrenages,

l.e moteur qui fait l'objet de la conférence de M. Courbier a pour but de donner par lu1-même une plus grande variation possible dans la vitesse propre du moteur et de permettre ainsi la suppression ou, tout au moins, la diminution du nombre des poulies des cônes ou des relais d'engrenages.

Le principe constste à farre varier le flux inducteur en augmentant ou en dimmuant l'entıefer. Pour cela, les pièces polaires sont ajustées a frottement doux dans le circuit, la surface de contact étant suffisamment grande pour que le joint magnétique ait une résıstance négligeable. L'écartement des noyaux est produit par une genouillière commandée par une tige filetée et une doulle écrou, munie d'un volant de mancuvre.

Un eifet analogue à celui produit par l'introduction de risıstances dans le circuit inducteur est obtenu ici en faisant varier la reluctance du.circuit magnétique par la modification de l'entrefer. La réaction d'induit diminuant à mesure que l'entrefer aưrmente, 1 en rusulte que le calage des balars reste fixe et que la production des étincelles du collecteur est reculée jusquà des écarts considérables de vitesse, tout en maintenant la puissance du moteur. Les courants induits er inducteurs restent donc constants, cest-à-dire que la constitution électrique du moteur est toujours la même, quels que soient les écarts variables des pôles, écarts limités seulement par la dispersion nuisible $d u$ tlux.

L'objection la plus sérieuse que l'on pouvait faire à ce gemre de moteurs était la diminution du rendement avec les grandes vitesses Pour y répondre. $M$. Courbier indique les résultats obtenus en talsant lessai, au frein de Prony, d'un moteur bipolaire de 28 ampères sous 220 volts.

Deux séries dic mesures ont été faites et correspondent à la pleine et à la demi-charge. Dans ces deux cas, l'entreter a varié de 3 à 48 millimetres et la vitesse de 485 à 1 Soo tours, Les rendements ont varié de 85 à 79 pour cent Jans la demi-charge et de 87 à 80 pour cent dans la pleine charge.

Un autre essai intéressant, fait sur la même machine, a été la sćnaration des pertes par la méthode de Housman et a domné de très bons résultats.

Comme conclusion de ces essais, l'on peut dire que le moteur à vitesses variables par pôles mobiles est, comme rendement et constitution electrique, en tous points comparable aux moteurs classiques; mais il présente, de plus, l'avantage de pouvoir donner des vitesses variant d'une façon continue et cela entre des limites tres étendues.

Au point de vue surtout de la continuité de la variation de vitesse, il peut rendre les plus grands services à la commande directe et individuelle des machines-outils.

\section{INVENTIONS NOUVELLES}

Nouveau régulateur de voltage des machines à courants alternatifs (Système Maurice Leblanc). - Brevet délivré le $22 \mathrm{mai}$ i go3 à la Société Anonyme Westinghouse, n" 329.187.

L'objet de l'invention est de réaliser un régulateur de voltage automatique, pour les alternateurs ou pour les machines d'induction employées comme génératrices, capable de faire varier, à chaque instant, l'intensité de leur courant d'excitation, de maniere à assurer la constance de leur voltage, quelles que soient les causes qui tendent à le faire varier: variation de debit ou de vitesse.

L'automaticıté est obtenue pardes moyens purement électriques et sans l'intervention d'aucun organe mécanique.

Ce régulateur est essentiellement constitué par la mise en opposition, dans un même circuit, d'une commutatrice développant cntre ses balais un voltage proportionnel à celui que l'on veut régler et d'une source d'électricité produisant un voltage constant, par exemple, une génératrice à courant continu; le courant qui circule dans le circuit de celte génératrlce et de la commutatrice traverse le circuit inducteur d'une seconde génératrice à courant contiuu servant de relais en reproduisant un courant d'intensité proportionnelle à celle du premier, mais fort amplifié, qui produit l'excitation de l'excitatrice à courant continu du ou des alternateurs ou des machines d'induction développant le voltage que l'on veut régler.

Pour constituer ce régulateur de voltage, le brever revendique l'application de machines à courant continu ayant un stator semblable à ceux des machines d'induction, muni en particulier d'un enroulement spécial monté en série dans le circuit principal de la 
machine, reproduisant, à la surface du stator, l'enroulement de l'armature et développant suivant toute direction une force magnétisante égale et de signe contraire à celle développée par l'armature, cela dans le but de constituer des machines à courant continu où l'énergie potentielle emmagasinée, pendant la marche, soit aussi petite que possible.

Enfin, en vue de l'application de ce régulateur au cas de surcompoundage des alternateurs ou des machines d'induction, les inventeurs se réservent la disposition d'un enroulement supplémentaire alımenté par le courant de l'excitatrice, sur l'inducteur de la génératrice de courant continu monté en oppositıon sur le circuit de la commutatrice et dont le voltage doit croitre avec le débit ou ce qui revient au mème, avec l'intensité du courant d'excitation des alternateurs ou machines d'induction.

Ce brevet dont le mémoire descriptif est très long contient une foule de détails sur le "dimensionnement» des apparells constituant le régulateur, sa mise en route et son mode de fonctionnement, sur les dispositions spéciales qu'il convient de donner à la commutatrice, aux machines à courant continu et à l'excitatrice, sur le réglage des machines d'induction, etc... Nous nous sommes donc borné: à indıquer le principe de l'ınvention et renvoyons au brevet lui-même les électriciens que ce mode de régulation intéresse plus spécialement

Procédé pour la fabrication de Ia cellulose de bois à l'aide du chlore dégagé dans l'électrolyse de chlorures métalliques. - Brevet du i 3 novembre 1902. C. Kellner, no 326.3 i 3 .

Ce procédé consiste à traiter d'abord les matières végétales avec un lait de chaux on une solution à $1 / 2$ pour 100 d'hydrate ou de carbonate alcalin, et à les soumettre ensuite à l'action du chlore gazeux provenant de l'électrolyse d'un chlorure métallique. Il faut avoir soin d'éliminer préalablement l'excès de liquide retenu par les matières végétales au cours du premier traitement, ce à quoi l'on parvient par leur essorage ou pressage.

Sous laction du chlore ainsi que sous l'influence de la chaleur résultant de l'absorption et de la fixation du chlore par les liquides contenus dans les matières végétales, il se produit une oxydation extrêmement énergique des lignines (matières incrustantes); cellesci pour certaines substances végétales sont transformées en matièrès completement solubles dans l'eau, tandis que pour d'autres, elles sont transformées en matières solubles dans des solutions alcalines diluées à 5 pour cent.

Si la transformation n'est pas complète par l'oxydation préalable, le résıdu qui, après traitement par l'eau, reste à côté de la cellulose et consiste en matières incrustantes oxydées et chlorées, peut être séparé de cette dernière substance, soit à l'arde d'un lait de chaux ou d'une solution très diluée d'hydrate ou de carbonate alcalin, soit à l'aide de ces deux traitements successifs à la température ordinaire ou à chaud, suivant la noture des substances végétales traitées; le résidu se dissout dans ces bains.

Dès que les matières végétales sont complètement imprégnées de chlore, il taut les retirer de l'appareil à chloruration, sans quoi la fibre végétale (cellulose) serait transformée en oxyccllulose et en composés de différentes teneurs en chlore qui, ne présentant pas de résistance, seraient sans applications pratiques. L'opération bien conduite donne un produit formé de cellulose presque pure. On la lave soigneusement á l'eau, on la blanchit dans certain cas, puis, une tois sèche, elle est prête à ètre livrée au commerce.

Grâce à ce procédé, la fabication de la cellulose peut donc être considérée comme un sous-produit de la fabrication des alcalis par électrolyse des chlorures alcalins. En ce qui concerne l'électrolyse des chlorures métalliques, le procédé permet de conserver totalement, ou du moins en majeure partie, à la cathode, les substances quı s'y forment, d'où $\mathrm{il}$ en peut résulter une application plus étendue dans certaines grandes industries chimiques.

\section{BIBLIOGRAPHIE}

Essal d'une théorie sur la propriété des Rivières non navigables ni flottables, par Luc Bernut, avocat, docteur en droit. Dijon, imprimerie Jobard, place Darcy, 9 .

Le livre de M. Bernot est court - il compte I 39 pages - mais il est exccllent et dénote, chez son auteur, une grande habitude d'un travail personnel qui ne sent pas la compilation et, au contraire, se complait dans la recherche des idées neuves.

Le mot proprété des Rivières qui sert de titre à l'ouvrage est révalateur - il réjouit les yeux de ceux quı connassent les aclmirables qualités du Code civil, fait et rédigé pour être pratique - il a dû navrer ceux qui préfèrent, en s'écartant des textes, professer un droit nouveau basé " sur le droit à la soif " du Coran Après avoir étudié les différents systèmes émis sur la propriété du lit que la loi de 1898 a attribuée cléfinitivement aux riverains, $M$. Bernot se demande quel est le droit de ceux-ci sur l'cau. C'est une "res nullius "! Telle est la réponse de la jurisprudence et de la doctrine. $M$. Bernot accepte cet adage, pour ce qu'il vaut ; c'est-à-dire plutôt pour une inexac- titude. C'est une "chose commune ", dit-il et dont l'usage est, par un texte formcl, réservé aux riverains (article 644) et encore cette cléfinition leur parait insuffisante. Puisque le lit de la rivière appartien aux rivcrains, peut-on séparer l'cau qui court sur le lit, de ce lit même arec lequel elle semble se confondre. Demolombe, écrivan bien avant la loi de 1898 , sans prendre partie pour le système de propriétć du lit qui de son temps n'était admis par personne, disait avec rasson que l'eau et le lit doivent appartenir à la même condition légale. Et M. Bernot conclut à la conséquence inévitable d'une conception juridique allant jusqu'à reconnaître une copropiété ındivise aux riverains, sur l'eau et par conséquent sur la pente qui en st la conséquence.

Et il nous met en garde contre les comparaisons faciles ct billantes mais peu exactes que certains partısans d'un système contraire ont multipliés avec une déplorable facilité. M. Salcilles, par exemple, s'est évertué à soutenir que l'eau cst essentiellement fugace, qu'une molécule liquide qui coule au droit de mon fonds, est liée à la molécule qui la suit, et qu'une pareille situation physique est incompatible avec un droit de propriété. Comparer l'eau au vent, devient un argument déjà vieilli, mais toujours répété. Il n'y a cependant aucune analogie réclle, répond $M$. Bernot entre l'air et l'onde; l'air ne fer jamais corps avec la terre sur laquelle il circule, comme l'eau ave lc sol qui la supporte: "Dira-t-on par excmple qu'une vallée est le lit du vent $"$ ?

Et si la rivıère non navigable est soumise à un droit d'indivision, plus fort même qu'un droit d'usage, presqu'un droit de copropriété soumis à un règlement d'utilité publique, il n'y a qu'un pas à faire pour admettre le système de la licitation qui permettra de préciser les droits de chacun et de les traduire en argent.

M Bernot le fait; et sans adhérer d'une façon absolument complète au projet de Grenoble, il en reconnaît la supériorité au point de vue juridique sur tous les autres systèmes proposés.

P. B.

Les lampes électriques à incandescence et leur appareillage, par M. E. Sartiaux, ingénieur, chef .des services électriques du Chemin de fer du Nord. - Fx. Baranger, éditeur, Paris.

M. E. Sartiaux nous donne, sous la forme d'une élégante brochure in-80 de 60 pages, illustrée de nombreuses gravures et photographies, le texte de la conférence qu'il a faite sur ce sujet, le 22 mars defnier, au Conservatoire National des Arts et Métiers. Hâtons-nous de dire que, quoique rédigée dans le langage simple qui convient aux personnes non familiarisées avec les termes de l'électrotechnique, cette brochure n'en intéressera pas moins bien des lecteurs plus sâvants par les détails pratiques qu'elle contient sur finstallation de l'éclairage domestique, au moyen des lampes à incandescence. Elle se recommande, d'ailleurs, de la haute compétence de son auteur dont les indications et les conseils seront écoutés avec profit.

Après un aperçu historique de l'éclairage privé depuis les temps prehistoriques jusqu'à nos jours, on y trouve, bien exposées, successivement, les questions de la pose des conducteurs électriques, des compteurs, des interrupteurs, coupe-circuits, doulles, porte-lampes prises de courant et appareillages divers. Puis on arrive à la fabrica-, tion des lampes à incandescence. Le principe de ces lampés é leups principaux modèles étant indiqués, $M$. Sartiaux décrit alors très clairement les procédés de fabrication du filament de carbone et de son support, le soufflage de l'ampoule, l'introduction du filament, les procédés pour faire le vide de la lampe, pour la vériffer et l'étalon* ner, la sceller sur son culot, etc. Viennent ensuite la description détaillée de divers systèmes, tel la lampe Nernst, et des indications très judicieuses sur l'emploi des lampes à incandescence, tant at point de vue de leur rendement lumineux que de leur durée et de leur consommation d'électricité.

\section{LIVRES NOUVEAUX EN FRANCE ET A L'ÉTRANGER}

Premiers principes d'électricité industrielle: piles, accumula: teurs, dynamos, transformateurs, par P. JANET, directeur de l'Ecole supérieure d'électricité, $5^{\circ}$ édit. In $-8^{\circ}$ avec fig. : $6 \mathrm{fr}$. Traité élémentaire d'électricité et de magnétisme, par G. Carey Foster et Alfred W. Porter. $2^{\theta}$ édit. In $-8^{\circ}$ (anglais), $4_{4} \mathrm{fr} 7^{5}$. L'Etablissement d'électricité dans les villes, par Osk. MmLer. $2^{\circ}$ livr. In-80 (allemand) : $25 \mathrm{fr}$.

Chemins de fer électriques, par Wilh. Kubler. $1^{\text {re }}$ année 1903. $4^{e}$ livr. Gr. in: $4^{0}$ avec fig. (allemand) : 22 fr. 25 .

Traité d'électro-métallurgie, par H. PONTHìre. 3e édit. In-8 avec fig. et I pl. en 4 couleurs : $15 \mathrm{fr}$.

La Prospection des Mines et leur mise en valeur, par M. Lecomir Denis, ing. civil des Mines, avec préface de M. Haton DE L Goupillierre. Beau vol. grand in. $8{ }^{\circ}$ de 552 pages avec nombreuses fig. Schleicher frères et Cie, éditeurs, Paris.

L'Imprimeur-Gérant: P. LEGENDRE. 